\title{
Simulation and analysis of the dynamics of a Fucus distichus (Phaeophyceae, Fucales) population
}

\author{
P. O. Ang Jr*, R. E. De Wreede \\ Department of Botany, University of British Columbia, Vancouver, British Columbia, Canada, V6T 1 Z4
}

\begin{abstract}
A general $9 \times 9$ matrix model based on recruit stages and plant size is proposed for a population of the brown alga Fucus distichus L. emend. Powell in False Creek, Vancouver, Canada. Twenty-six matrices were constructed each representing a monthly time interval covering the period from July 1985 to November 1987. Yearly matrices were also constructed as a product of monthly matrices covering periods of $1 \mathrm{yr}$. The characteristics of these matrices were evaluated by comparing the dominant eigenvalue (i.e. the population growth rate, $\lambda$ ), the stable distribution, and reproductive values for each matrix. The relative contribution of each matrix parameter to population growth was assessed using elasticity analysis. The survival and transition of the plants among size classes contribute at least $50 \%$ to the population growth rate. However, because $F$. distichus plants do not exhibit vegetative regeneration, the population can only experience positive growth in the presence of recruitment. Absence of a germling bank has little effect on monthly projected population growth, but can reduce the population growth rate projected from the yearly matrix by as much as $83 \%$. Current population size structure is very different from the projected stable distribution. The stable distribution generally has a large proportion of the plants in the recruit stage. Reproductive values are positively related to plant size but are comparable among the largest 4 size classes. Random combinations of monthly, seasonal, or yearly matrices were used to simulate population growth under various fluctuating environmental conditions. A negative growth rate was obtained in $>60 \%$ of the simulation runs. In reality within the sampling period covered, the population is on the decline. It is likely that this population may recover by occasional pulses of a large number of recruits, as was observed in 1986 .
\end{abstract}

\section{INTRODUCTION}

Mathematical models are tools used to provide insight into the observed natural patterns of various populations. They can help point out gaps in our understanding of the dynamics of these populations and hence suggest future directions for research. Attempts to model algal populations were often associated with the need to exploit algae as a resource and to provide a basis for sound management of this resource. Several approaches have been made, each utilizing different types of models. The seasonal growth of the giant kelp Macrocystis pyrifera (L.) C. Ag was modelled by Anderson (1974). Silverthorne (1977) developed an optimal harvesting strategy for

\footnotetext{
- Present address: Fisheries Research Laboratory, Fisheries and Oceans Canada, PO Box 550, Halifax, Nova Scotia, Canada B3J 2S7
}

Gelidium robustum (Gardn.) Hollenberg \& Abbott. Seip (1980) used a logistic model to evaluate the optimal harvesting for Ascophyllum nodosum (L.) Le Jol. A stochastic model was utilized by Smith (1986) to predict the outcome of annual harvesting on the interaction between Laminaria longicruris De la Pylaie and L. digitata (Hudson) Lamouroux and on the yield of their biomass using different harvesting strategies. Jackson (1987) modelled the growth and biomass yield of M. pyrifera. Ang (1987) and Ang et al. (1990) used projection matrix models to assess harvesting strategies for Sargassum spp. and Iridaea splendens (Setchell \& Gardner) Papenfuss respectively.

In recent years, models were also developed to evaluate the basic biology and ecology of algal populations. Jackson et al. (1985) assessed the morphological relationships among fronds of Macrocystis pyrifera. Nisbet \& Bence (1989) developed a family of models depicting a simplified relationship between 
juvenile recruitment and adult population density of this same species, incorporating a recruitment inhibitory factor represented by a random variable. They found that despite the overly simplified assumptions in their models, they were able to produce patterns of fluctuation in the population density very similar in range to those observed in nature. Their models indicate that factors affecting recruitment may be very important in determining the dynamics of the giant kelp population. Burgman \& Gerard (1990) developed a more comprehensive model of $M$. pyrifera, incorporating life history stages, environmental and demographic stochasticity, and density-dependent interactions. Their model can predict the monthly changes in the density of each stage of the population for up to $20 \mathrm{yr}$. Demographic characteristics of other species of algae have also been modelled, e.g. De Wreede (1986) used a matrix model to evaluate changes in the age class distribution of Pterygophora californica Ruprecht. Similarly, Áberg (1992a, b) used a matrix model to assess and simulate changes in the vital rates of 2 populations of Ascophyllum nodosum in different environments exposed to annual variation of winter ice cover.

In a previous paper (Ang \& De Wreede 1990), we presented matrix projection models based on size and stage that can be used with algae having different basic types of life histories. Matrix models for Sargassum siliquosum J. Ag. and Laminaria longicruris were developed to show the utility of these models. Using these models, one of the questions that can be asked about the population is what would happen to the population if the conditions defined by the matrix remain unchanged? By comparing the 'fates' of the population subjected to the constraints of different matrix elements, the significance of the different transition probabilities experienced by the population at different times can be evaluated. Furthermore, elasticity analysis can be used to assess the relative contribution of each matrix element to population growth rate (de Kroon et al. 1986).

In this paper, a general matrix model based on recruitment stages and plant size is developed for the Fucus distichus L. emend. Powell population in False Creek, Vancouver, British Columbia, Canada. Transition matrices and their products were constructed to represent the behaviour of the population in monthly, seasonal and yearly time intervals. Based on these models, the importance of recruitment and the relative contribution of each matrix parameter to population growth rate were assessed. To simulate different fluctuating environments conceivably faced by the population, random combinations of monthly, seasonal, and yearly matrices were generated and the effect of these on population growth rate evaluated.

\section{THE POPULATION OF FUCUS DISTICHUS}

The population of Fucus distichus in False Creek, was monitored monthly from July 1985 to November 1987 within 3 permanent quadrats $\left(0.25 \mathrm{~m}^{2}\right)$ for patterns of recruitment, age- and size-dependent reproduction, growth and mortality. Using log linear and association analyses, age and size were both found to be statistically significant as descriptors of these demographic parameters, but size was more important than age (Ang 1991a, b).

Reproductive plants were found throughout the sampling period, but peaked in fall and winter of each year. However, peaks in recruitment of microscopic germlings (<1 mm microrecruits) were not always followed by peaks in the recruitment of macroscopic plantlets (macrorecruits) within the permanent quadrats. This may be due to a differential survivorship of microrecruits over time or to the possible existence of a 'germling bank'. Patterns of survival and emergence of macrorecruits may be independent of those of microrecruits or may be unrelated to the prevailing reproductive phenology. More detailed information on various phenomena related to the demographic parameters of this population are given in Ang (1991a, b, 1992) and Ang \& De Wreede (1992), as well as in the relevant sections below.

\section{THE MODEL}

Fucus distichus plants exhibit only a sporophytic phase of the life history (Bold \& Wynne 1985). The dynamics of the $F$ distichus population can thus be appropriately described by a monophasic matrix model (Ang \& De Wreede 1990). Detailed monthly monitoring of the fate of individual plants within the permanent quadrats provided the basic information on the dynamics of the population in False Creek. Twenty-six matrices were constructed each representing a transition period of 1 mo (except for one representing the 2 mo period from July to September 1985 and another one, from October to December 1986). Thus, the total sampling period covered from July 1985 to November 1987 was 28 mo.

Plants were divided into 6 size classes, with Size Class $1 \quad<1 \mathrm{~cm}$ in length) representing the macrorecruits. No plant $<4.5 \mathrm{~cm}$ in length was found to be reproductive (Ang 1991a), so all plants $\geq 1$ and $<4.5 \mathrm{~cm}$ in length were grouped as Size Class 2. All the remaining plants were grouped in size classes with equal size class interval of $5 \mathrm{~cm}$, with the exception of Size Class 6 which included all plants $>19.5 \mathrm{~cm}$ in length. In addition, data on settling blocks set out in the field provided information on the recruitment of the microrecruits, as distinguished from the recruitment of 
macrorecruits into the permanent quadrats (Ang 1991a). There was generally a 4 mo lag between the release of gametes by the reproductive plants and the time when the germlings became visible macrorecruits (Ang \& De Wreede 1992). Hence, 3 recruitment stages (Microrecruit Stages 1, 2 and 3) were represented in each matrix to account for this transition, with a fourth stage equivalent to macrorecruits in Size Class 1 . The basic $9 \times 9$ transition matrix is therefore more complex than the monophasic matrix described in Ang \& De Wreede (1990) and is of the form:

$\mathbf{M}=\left[\begin{array}{ccccccccc}0 & \cdot & \cdot & . & 0 & F_{3} & F_{4} & F_{5} & F_{6} \\ R_{1} & 0 & \cdot & . & \cdot & \cdot & \cdot & \cdot & 0 \\ 0 & R_{2} & G & 0 & 0 & 0 & 0 & 0 & 0 \\ 0 & 0 & R_{3} & P_{11} & P_{12} & . & & \cdot & P_{16} \\ \cdot & & R_{4} & P_{21} & & & & & \cdot \\ \cdot & & \cdot & \cdot & & & & & \cdot \\ \cdot & & \cdot & \cdot & & & & & \cdot \\ \cdot & & \cdot & \cdot & & & & & \cdot \\ 0 & . & 0 & P_{61} & . & . & . & \cdot & P_{66}\end{array}\right]$

where $R=$ the probability of moving from one recruitment stage to the next, with $R_{3}$ and $R_{4}$ representing respectively the probability of microrecruits becoming visible macrorecruits $<1 \mathrm{~cm}$ (i.e. Size Class 1 ) or $\geq 1$ to $<4.5 \mathrm{~cm}$ in size (i.e. Size Class 2); $G=$ the probability of staying as microrecruits, i.e. of staying in the germling bank; $F=$ the fecundity value for each size class; and $P=$ the probability of moving from one size class to the other. Where a transition is not possible, it is represented by a 0 .

As indicated by the dotted lines, the transition matrix $\mathbf{M}$ can be partitioned into 4 submatrices as:

$$
\mathbf{M}=\left[\begin{array}{ll}
\mathbf{A} & \mathrm{C} \\
\mathbf{B} & \mathrm{D}
\end{array}\right]
$$

where $\mathbf{A}$ describes the transition among the recruits, and $\mathbf{B}$, the transition from microrecruits to macrorecruits in Size Class 1, as well as to larger size classes. $\mathrm{C}$ describes the fecundity, i.e. the contribution of larger plants to microrecruits, and $\mathbf{D}$, the transition among plants in different size classes.

The basic column vector represents the distribution of the individuals in the different recruitment stages and size classes and is given as a matrix

$$
\mathbf{N}=\left[\begin{array}{l}
\text { Microrecruit Stage } 1 \\
\text { Microrecruit Stage } 2 \\
\text { Microrecruit Stage 3 } \\
\text { Size Class } 1(<1 \mathrm{~cm}) \\
\text { Size Class } 2(1-<4.5 \mathrm{~cm}) \\
\text { Size Class } 3(4.5-<9.5 \mathrm{~cm}) \\
\text { Size Class } 4(9.5-<14.5 \mathrm{~cm}) \\
\text { Size Class } 5(14.5-<19.5 \mathrm{~cm}) \\
\text { Size Class } 6(\geq 19.5 \mathrm{~cm})
\end{array}\right]
$$

Growth of the population is then defined by:

$$
\mathbf{N}_{t+1}=\mathbf{M} \mathbf{N}_{t}
$$

where $t$ is time.

\section{PARAMETER ESTIMATION}

\section{Transition among the size classes $(P)$}

Any plant (macrorecruit) in any size class can become longer within a time period $t$ to $t+1$ due to growth and move to a larger size class, or become shorter due to die back or degeneration of receptacles or terminal branches and move to a smaller size class. The plant may also stay in the same size class if growth or die back is not large enough.

Individual plants in the permanent quadrats were mapped separately and measured each month from the base of the holdfast to the tip of the longest branch. They were sorted into 6 size classes defined in the column vector $\mathbf{N}$. Their 'fate' over each month was scored according to whether they survived and, if they did, whether they became larger, smaller or showed no change in size. The probability of moving into different size classes was then calculated to give the elements $P_{1 j}$. A separate matrix was constructed for each quadrat for each month. The mean of each transition probability of the 3 matrices for each month was calculated and used as the submatrix $\mathbf{D}$ of the final monthly matrix $\mathbf{M}$.

\section{Fecundity $(F)$}

Plants in Size Classes 1 and 2 never became reproductive (Ang 1991a), their fecundity values were therefore always nil. For reproductive plants in larger size classes, the potential number of eggs produced by the plant is size-dependent, but the actual number produced is not. The former was measured as the number of eggs found in the conceptacle per unit area of the receptacle. The latter was demonstrated based on the number of egg clusters released from receptacles of known sizes (Ang 1991a). The number of eggs (zygotes) entering the population as new recruits is therefore independent of the plant size. Fecundity values for plants in the other 4 size classes can be calculated in terms of the mean number of microrecruits recorded on settling blocks every month $(R)$. The number of microrecruits produced per reproductive plant $(Z)$ per month is given as:

$$
Z=\frac{R}{\sum\left(W_{i} n_{i}\right)} \quad \text { for } i=3,4,5,6
$$

where $W_{1}$ is the mean proportion of reproductive individuals, and $n_{j t}$ the mean number of individuals in each 
size class $i$. The fecundity value $F_{i}$ for each size class $i$ is therefore calculated as:

$$
F_{i}=Z W_{11}
$$

with $F$ being a function of the mean probability of the individual plants being reproductive.

The actual number of microrecruits was recorded only starting in November 1985. The numbers of microrecruits for September and October 1985 were calculated as a mean of the number of microrecruits of the same months in the following 2 yr. The number of microrecruits in July 1985 was back-calculated based on the data of macrorecruits in November 1985. The mean number of microrecruits for July 1986 and 1987 was too small to account for the number of macrorecruits in November 1985, hence could not be used to fit the model. Between April and August 1986, and July and August 1987, no microrecruits were recorded in the settling blocks (Ang 1991a). In order to maintain the irreducibility of the transition matrices representing these months (i.e. to avoid having one row of the matrices with all 0 values), fecundity for each of the 4 size classes was given a very small value of $1 \times 10^{-10}$.

\section{Transition among microrecruits $\left(R_{1}\right.$ and $\left.R_{2}\right)$}

Microrecruits at the initial stages survived better at higher density, although this positive densitydependence is relatively weak (Ang \& De Wreede 1992). Twenty-two percent of the microrecruits survived the first 2 mo after being seeded in the density blocks in November 1986 (Ang \& De Wreede 1992). Estimates of microrecruit survivorship $\left(R_{1}\right.$ and $\left.R_{2}\right)$ at different time periods were based on a factor of $22 \%$, taking into account the positive effect of density.

Since only the number of microrecruits, based on the settling blocks, and that of macrorecruits, based on the permanent quadrats, were known from field observation, the number of recruits between these stages (i.e. Microrecruit Stages 2 and 3) can only be hypothetical values. The number of Stage 3 microrecruits in July 1985 was set at 1000 . This is approximately the smallest value that can account for the number of macrorecruits recorded in the permanent quadrats in September 1985 , given a microrecruit survivorship of approximately $3 \%$ (Ang 1991a). The number of Stages 2 and 3 microrecruits in the subsequent months was calculated based on the estimated survivorship for each stage.

\section{Transition from micro- to macrorecruits $\left(R_{3}\right.$ and $\left.R_{4}\right)$}

It is assumed that only a Stage 3 microrecruit can become a macrorecruit. This transition probability was estimated as a ratio between the number of Stage 3 microrecruits and the number of macrorecruits. A few macrorecruits were $>1 \mathrm{~cm}$ when first detected, so that it is possible for a Stage 3 microrecruit to become a member of Size Class 2 within a single transition period.

\section{Germling bank $(G)$}

The presence of a germling bank was suggested in Ang (1991a) to account for the continuous presence of macrorecruits in the permanent quadrats throughout the year despite the absence of any microrecruits in summer. As Microrecruit Stages 1 and 2 are monthly stages, they would have to move on to an older stage in every monthly time step. Microrecruits could thus stay in the germling bank only after they reach Stage 3 . The probability of staying in the germling bank was estimated based on a factor of $22 \%$, similar to that used in the estimation of the probability of transitions from $R_{1}$ to $R_{2}$ and $R_{2}$ to $R_{3}$.

\section{METHODS OF ANALYSIS AND SIMULATION}

Construction of the transition matrices is an attempt to describe the dynamics of the population as realistically as possible in mathematical terms. Both the elements of the submatrix $\mathbf{D}$ and those of the column vector are mean values estimated from the 3 quadrats and, therefore, have variances around their respective means. However, fecundity values, transitions among microrecruits, and transitions from micro- to macrorecruits cannot be estimated from the 3 permanent quadrats separately. All 3 quadrats were located within the same population and highly localized recruitment confined only within each quadrat, while possible, was unlikely. Furthermore, the micro-recruitment data on the settling blocks represented recruitment for the whole population, and not for any particular quadrat. Macrorecruitment data, on the other hand, were based on individual quadrats. Under these constraints, elements of submatrices $\mathbf{C}$ and $\mathbf{D}$ were assumed to be fixed for each matrix. Transition values for the microrecruits and those from micro- to macrorecruits were then fine-tuned in an iterative manner in order to achieve a resulting population distribution within $\pm 1 \mathrm{SE}$ of the mean value of the actual distribution estimated from the 3 quadrats. This was largely successful except for the months of May to June and June to July 1986 where the simulated population size is much larger than that of the actual field population (Fig. 1, Total). This discrepancy is mainly due to a larger simulated Size Class 1 population over this time period (Fig. 1, Size Class 1). Except for this dis- 


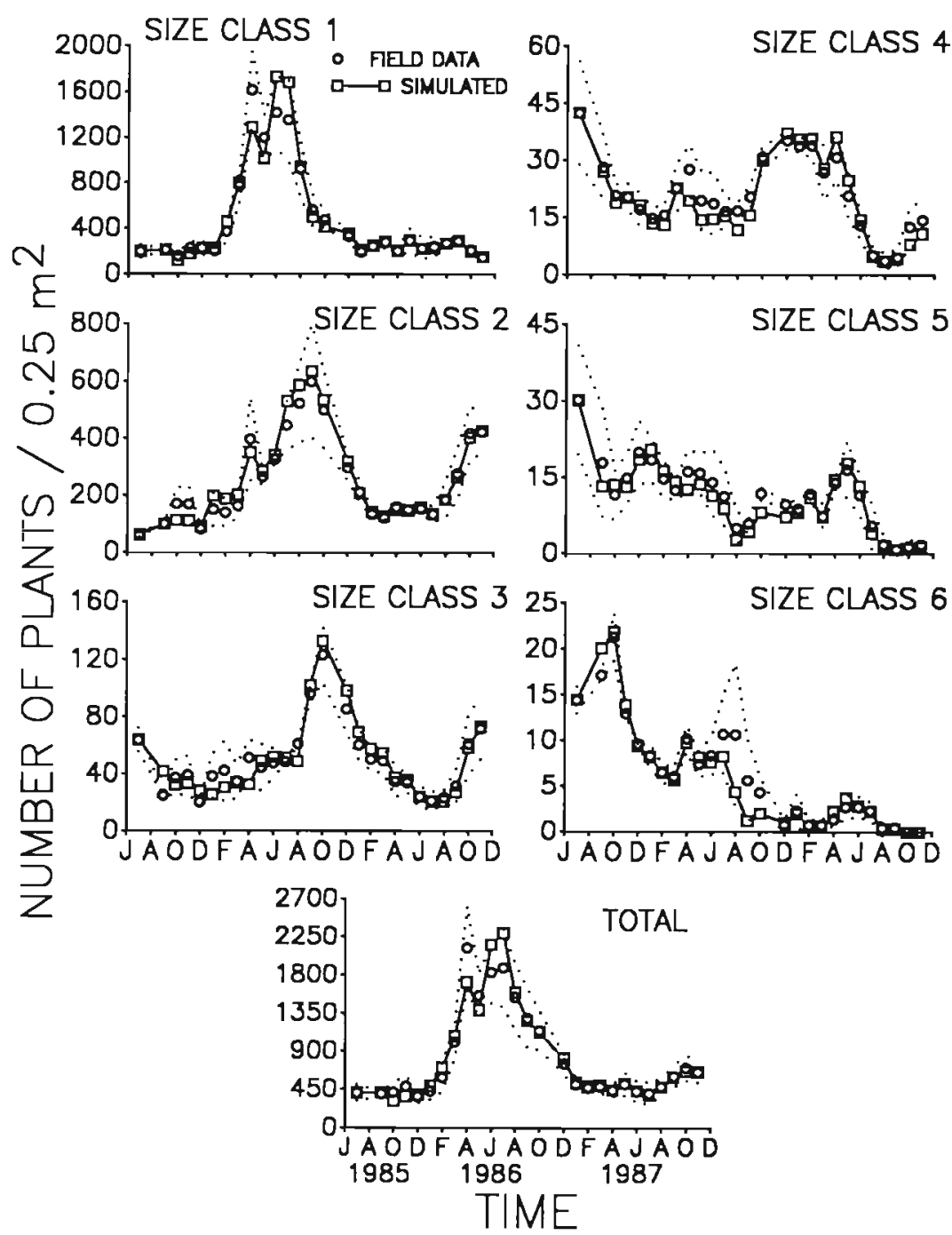

Fig. 1 Fucus distichus. Mean no. of plants in each size class and total no. of plants recorded from field observations over time, compared with the number simulated from the matrix models. Dotted outlines indicate the upper and lower boundaries of $1 \mathrm{SE}$ of the field estimates

crepancy, these monthly matrices project the distribution of this Fucus distichus population relatively well and further analyses can be made on these matrices to understand the dynamics of this population. A complete listing of all the monthly matrices and monthly size class distribution of the population is given in Ang (1991c). These matrices represent information on a total of 58402 observations made over the sampling period.

\section{Analysis of the model}

\section{Monthly matrix}

A population undergoing changes as defined by a transition matrix will eventually grow at a constant growth rate $(\lambda)$. This growth rate can be calculated as the dominant eigenvalue of the transition matrix (Leslie 1945, Caswell 1989). Matrix $\mathbf{M}$, which includes all the matrix parameters (i.e. $P, F, R$ and $G$ ), can be compared with submatrix $D$, which includes only the matrix parameter $P$. The dominant eigenvalue for matrix $\mathbf{M}$ estimates the overall population growth rate, that for submatrix $D$ the population growth rate due to the survival and transition of plants in the size classes $(P)$ alone. By comparing the 2 eigenvalues, the relative effect of recruitment and reproduction versus survival of plants on population growth can be assessed. The dominant eigenvalue was calculated for each monthly $\mathbf{M}$ and $\mathbf{D}$ to evaluate differences in the population growth rate at any point in time. It was also calculated for each monthly matrix with $G$ set to 0 . This is to assess the relative effect of the absence of a germling bank on the population growth. A dominant eigenvalue $>1$ indicates that the population is growing, a value $<1$ indicates that the population is declining. An eigenvalue $=1$ suggests that the population is neither increasing nor decreasing.

Elasticity analysis (de Kroon et al. 1986) measures the relative contribution of each matrix entry to population growth. The sum of all contributions equals 1 . This technique was used to assess the relative contribution of each matrix parameter to population growth at each month, i.e. the proportional sensitivity of population growth rate to each matrix entry.

When the population is growing at a constant growth rate $(\lambda)$, a stable distribution characterized by a constant relative proportion of each recruit stage and size class is attained. The stable distribution is the right eigenvector corresponding to the dominant eigenvalue of each matrix (Caswell 1989). The stable distribution for each monthly matrix $\mathbf{M}$ was calculated and then compared, using a chi square-test, with the observed distribution of the plants in the field to assess the relative stability of the plant distribution. The reproductive value for each monthly matrix $\mathbf{M}$ was also calculated. The reproductive value is the left eigenvector corresponding to the dominant eigenvalue (Leslie 1948 , Caswell 1989). It is a measure of the relative contribution or importance of each recruit stage or size class to future generations of the population. 


\section{Yearly matrix}

In this Fucus distichus population, macrorecruits were present every month throughout the sampling period (Fig. 11 in Ang 1991a). In each month and thereafter, the conditions that these new individuals were facing would conceivably be different from those faced by other individuals recruited at other months. If these individuals were assumed to grow on a yearly cycle, the dynamics of growth and mortality that they experienced can be assessed using a yearly matrix.

A yearly matrix $\mathbf{Y}$ was constructed by obtaining the product of monthly matrices corresponding to a period of 1 yr, e.g. July 1985 to July 1986. Starting from July 1985 and then each month subsequently, 16 yearly matrices were obtained. The last yearly matrix covers the period from December 1986 to November 1987, the end of the sampling period. A complete listing of the yearly matrices is given in Ang (1991c).

The dominant eigenvalue of each yearly matrix, as well as that of the product of the submatrix $\mathbf{D}_{Y}$ corresponding to a period of $1 \mathrm{yr}$, was calculated. In addition, the eigenvalue of each yearly matrix, derived from monthly matrices with $G$ set to 0 , was also calculated. These different eigenvalues were compared. Elasticity analysis, stable distribution and reproductive values of each yearly matrix were also calculated.

\section{Simulation and projection}

The product of all the monthly matrices is a matrix $\mathbf{T}$ describing the transition of the population over the whole sampling period from July 1985 to November 1987. The population may be projected to grow following the parameters defined by this matrix. Population growth rate over the 28 mo sampling period is then given by the dominant eigenvalue of matrix $T$. This assumes that population growth follows a cycle defined invariably by the 28 mo period sampled. This undoubtedly represents only one of the possible patterns that may be exhibited by the population. Other possible patterns of growth may be defined by the monthly, seasonal, or yearly matrices. These patterns may be simulated by a randomized combination of different individual matrices or matrices grouped in different seasons or years and projected over a fixed period of time.

\section{Randomized monthly matrices}

Within the sampling period, there were 2 complete 12 mo cycles covering the period from November 1985 to November 1987 . Each month within a 12 mo cycle can therefore be represented by 2 matrices. To project the growth of the population, 1 of the 2 matrices was selected at random. The chronological sequence of the
12 mo cycle from November to November was not changed. The growth of the population was projected over a 60 mo period $(5$ yr) 100 times. The growth rate for each resulting matrix was calculated, and the mean growth rate of these 100 replicates, the standard deviation, and the highest and lowest growth rates were recorded.

This approach is conservative in the sense that at any one month, the dynamics of the population would be defined only by one of the 2 matrices. These matrices may not represent the effect of the full range of environmental extremes that the population may conceivably face in nature.

\section{Randomized seasonal matrices}

The dynamics of population growth may be related to seasonality. To simulate this, monthly matrices were grouped to represent the 4 seasons. Each seasonal matrix $\mathbf{S}$ is a product of 3 monthly matrices, e.g. a winter matrix is represented by the product of December to January, January to February and February to March matrices. Given that 2 complete years were covered by the sampling period, there are 2 matrices representing each season. Either 1 of the 2 matrices for each season was selected at random following the seasonal sequences of winter, spring, summer and fall. The growth of the population was projected over 5 yr ( 20 seasons) and 10 yr (40 seasons), each replicated 100 times. The growth rate for each resulting matrix was calculated and the mean, standard deviation, and highest and lowest growth rates among the replicates were recorded.

\section{Randomized yearly matrices}

The population was simulated to grow following yearly cycles. Yearly matrices are products of 12 monthly matrices. Each of the 2 yearly matrices was selected at random and the population simulated to grow following the combinations of these yearly matrices. The growth of the population was projected for 5 and $10 \mathrm{yr}$, each replicated 100 times. The growth rate for each resulting matrix was likewise calculated and the mean, standard deviation, and the highest and lowest growth rates among replicates were recorded.

\section{RESULTS}

\section{Analysis of the model \\ Monthly matrix}

Field data suggested that plants in Size Class 1 genexally had a lower survivorship than those in other size classes (Fig. 2). Most plants suffered a greater mor- 


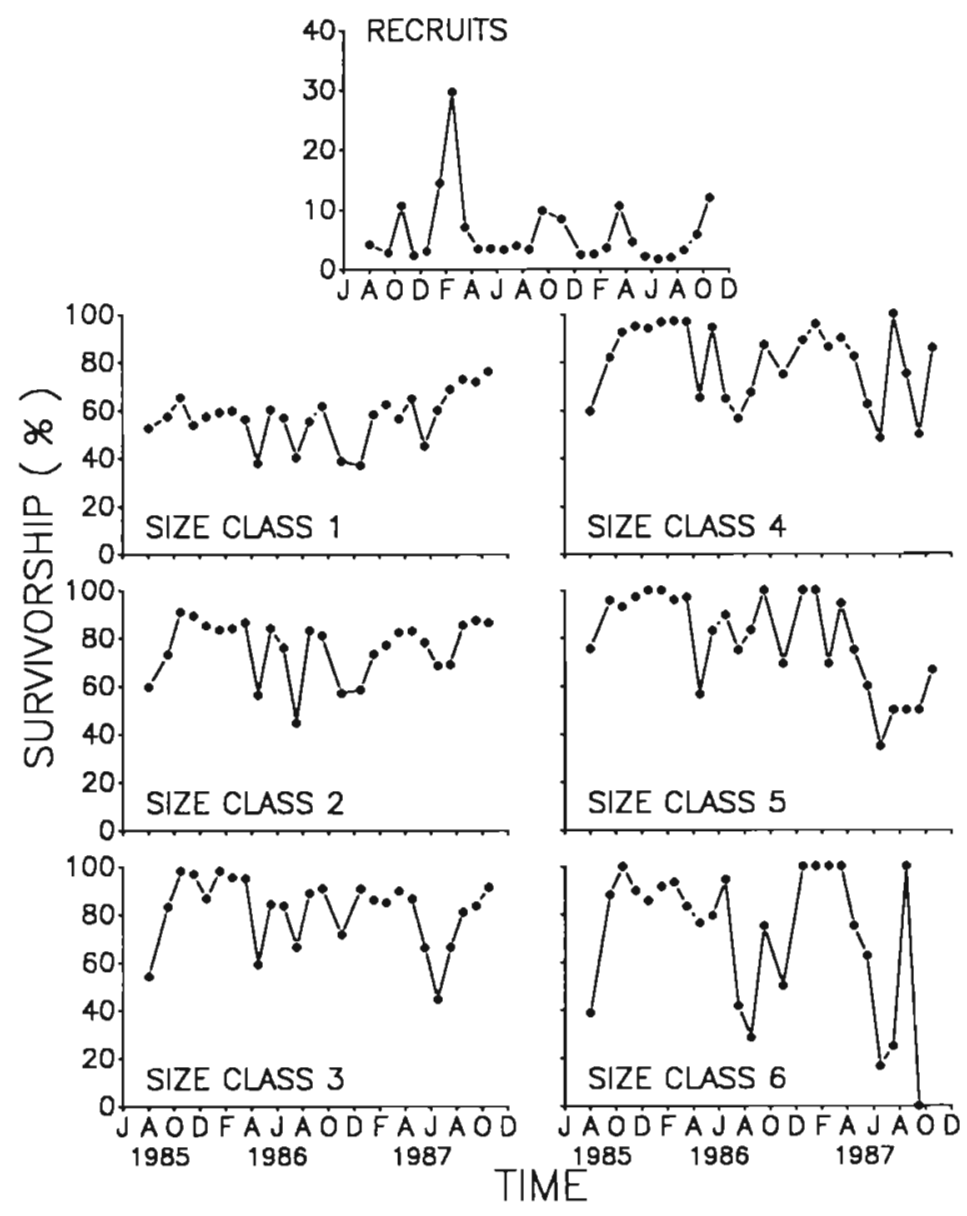

Fig. 2. Fucus distichus. Mean survivorship of plants in different size classes over time as estimated from field observations

tality during spring and summer 1986 than during fall and winter 1986. Plants in Size Classes 3 to 6 also showed a high mortality in summer 1987, but this pattern was less distinct for smaller plants. Plants in Size Class 6 suffered consistently high mortality (>60\%) in all 3 summers from 1985 to 1987.

Patterns of plant survival are reflected in the submatrix D. Fucus distichus plants do not exhibit vegetative reproduction, i.e. regeneration from the holdfast was not observed (Ang 1991a). Hence, if the population were to grow under the conditions described by $\mathbf{D}$ alone, the population would not be able to maintain itself. This is indicated by the small $(<1$ ) eigenvalues for most of the monthly submatrices D (Fig. 3). In the few cases where the eigenvalue $\approx 1$, it is due to the high survivorship of larger plants, notably plants in Size Class 6 . With reproduction and recruitment, the increase in population growth rate is not always large enough to prevent the decline of the population. However, in some instances, e.g. September to October 1986, the population experienced a sharp increase in growth rate (Fig. 3). At very low fecundity (late spring 1986 and summer 1986 and 1987), population growth rate would be dependent on the survival of the plants alone. The absence of a germling bank has either no effect or would lower the population growth rate by only 1 to $4 \%$.

Results of the elasticity analysis indicate that survival and transition among plant size classes are the most important parameters and contribute at least $50 \%$ to the population growth rate at any time (Fig. 4). Other parameters like survival and transitions among microrecruit stages and from micro- to macrorecruits, germling bank and fecundity are also important in some months. The relative contribution from these parameters generally follows a similar pattern, being lower in early winter 1985 and 1986, and in late spring to mid summer 1986 and 1987 than in the rest of the year. In general, the germling bank contributes the least to population growth (Fig. 4).

Plants in Size Classes 1 and 2 never became reproductive, so their contribution to population growth rate was only through their survivorship. This contribution could be very large $(>60 \%)$ at times. The contributions of reproduction from the other size classes are comparable and are usually small. There is a lot of variation in the way different size classes contribute to population growth. In a few cases, the contribution is mainly from a single size class (e.g. January to February 1987 from Size Class 5). These are the times when very low mortality was experienced by that particular size class.

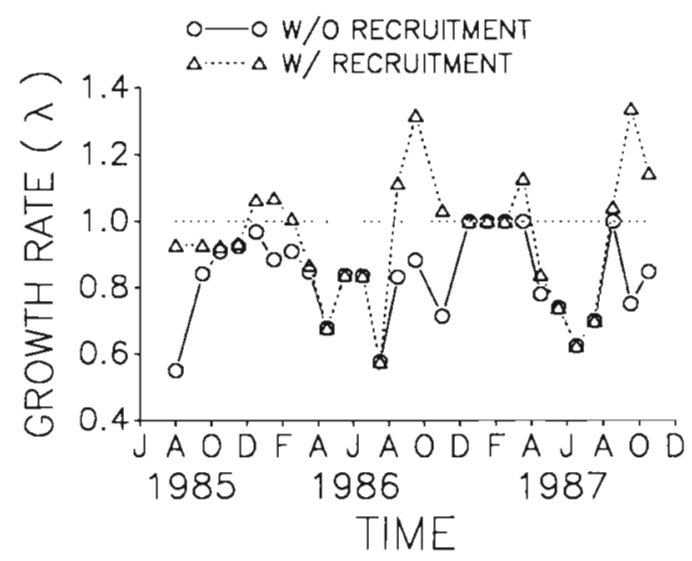

Fig. 3. Fucus distichus. Dominant eigenvalue (population growth rate) calculated for each monthly matrix $\mathbf{M}$ (with recruitment) and monthly submatrix $\mathbf{D}$ (without recruitment). Dotted horizontal line indicates eigenvalue $=1$ 


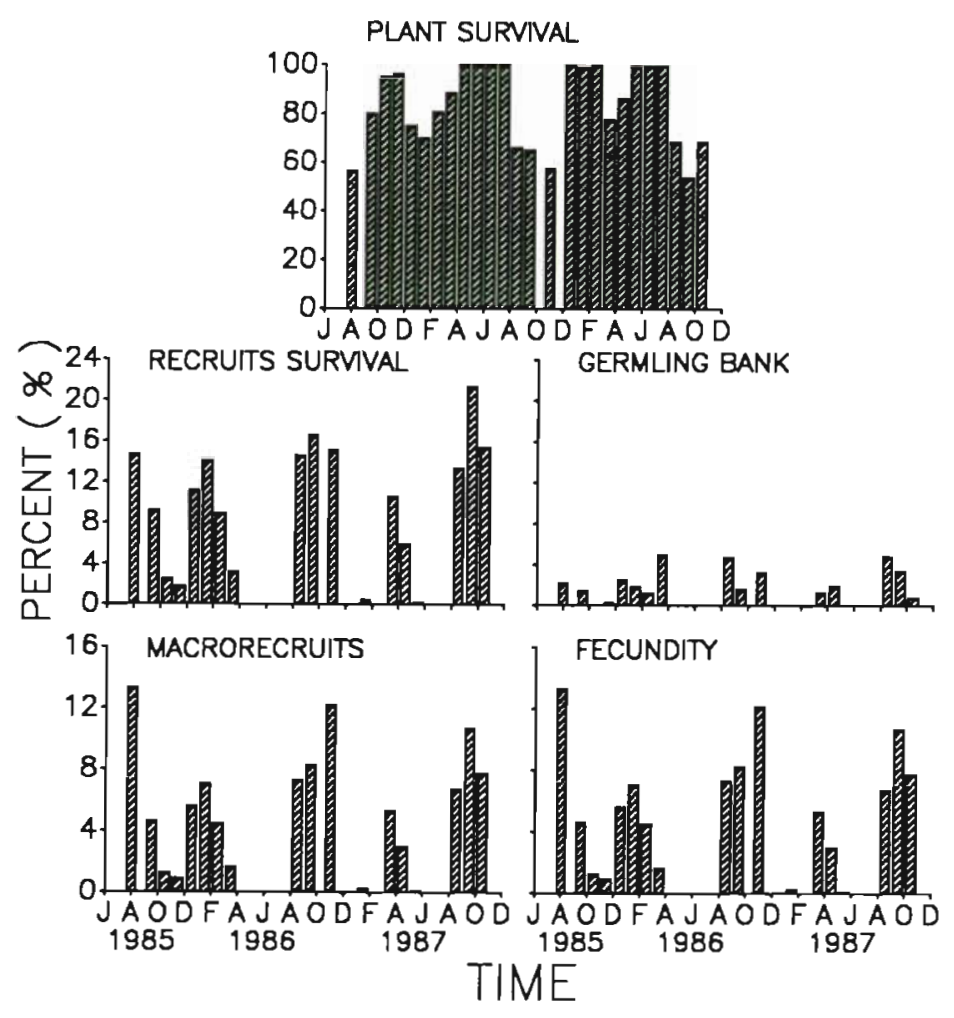

Fig. 4. Fucus distichus. Relative contribution (\%) of different matrix parameters in each monthly matrix $\mathbf{M}$ to population growth rate, as estimated from elasticity analysis. Matrix parameters are plant survival: survival and transition among plants in Size Classes 1 to 6 ; recruits survival: survival and transition among microrecruit stages; macrorecruits: survival and transition from micro- to macrorecruits; germling bank: probability of staying in the germling bank; and fecundity: fecundity of Size Classes 3 to 6

When the population was projected to grow based on the estimated matrix elements and attained a stable distribution, a very high proportion of the plants would be in the form of recruits, e.g. as high as $99 \%$ in December to January 1987 (Fig. 5). The stable distribution would have more large plants than recruits only when fecundity was low or negligible.

The number of recruits projected in the stable distribution included those recruits of intermediate stages. There are no actual field data on Microrecruit Stages 2 and 3. Hence, when comparing the projected stable distribution of the plants in the size classes with the observed size class distribution, it is more appropriate to compare only the pattern of distribution of the macro-plants (Size Classes 1 to 6) rather than that of all the size classes including the recruits. In all cases, the projected stable distribution is very different from the distribution recorded from field observations (chisquare test, $p<0.001$ ).

Size Class 6 usually has the highest reproductive value. The extreme high value $\left(>10^{3}\right)$ in February to March and August to September 1987 is related to its high survival rate $(\approx 1)$. From summer 1985 to fall 1986, reproductive values of the 4 largest size classes are comparable. The total reproductive values are positively related to population growth rate.

\section{Yearly matrix}

Transitions among the recruits and across different size classes are possible in a yearly matrix, hence all the matrix elements are nonzero. Because of the absence of any vegetative reproduction, initial populations recorded at different months in 1985 to 1986 would decline rapidly if they were to be maintained by the survivorship of the plants alone. In $50 \%$ of the cases, reproduction and recruitment only slowed down the rate of decline. In other cases, i.e. July 85-July 86, October 85-October 86 to December 85-December 86, May 86-May 87 and June 86-June 87, the population growth rate became positive in the presence of reproduction and recruitment. In the absence of a germling bank, all of the populations would decline and experience from 45 to $83 \%$ reduction in the growth rate.

There is a discernible seasonal pattern in the relative contribution of the different demographic parameters to population growth within each year (Fig. 6). In general, survival of microrecruits contributed the least to population growth rate, though it could be more important than the germling bank in matrices beginning in spring. Certainly, it contributed the most to growth rate in the April 86-April 87 matrix.

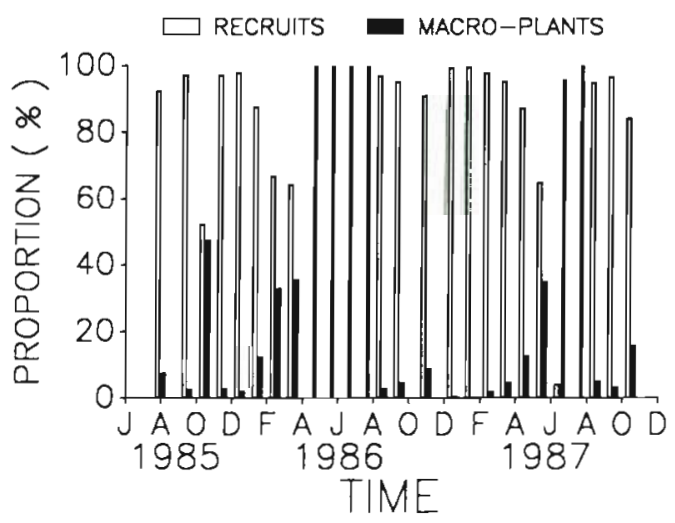

Fig. 5. Fucus distichus. Proportion (\%) of all recruits versus macro-plants in the size classes at stable distribution calculated for each monthly matrix $\mathbf{M}$. Recruits included all intermediate stages of microrecruits and macro-plants included all plants from Size Classes 1 (with macrorecruits) to 6 


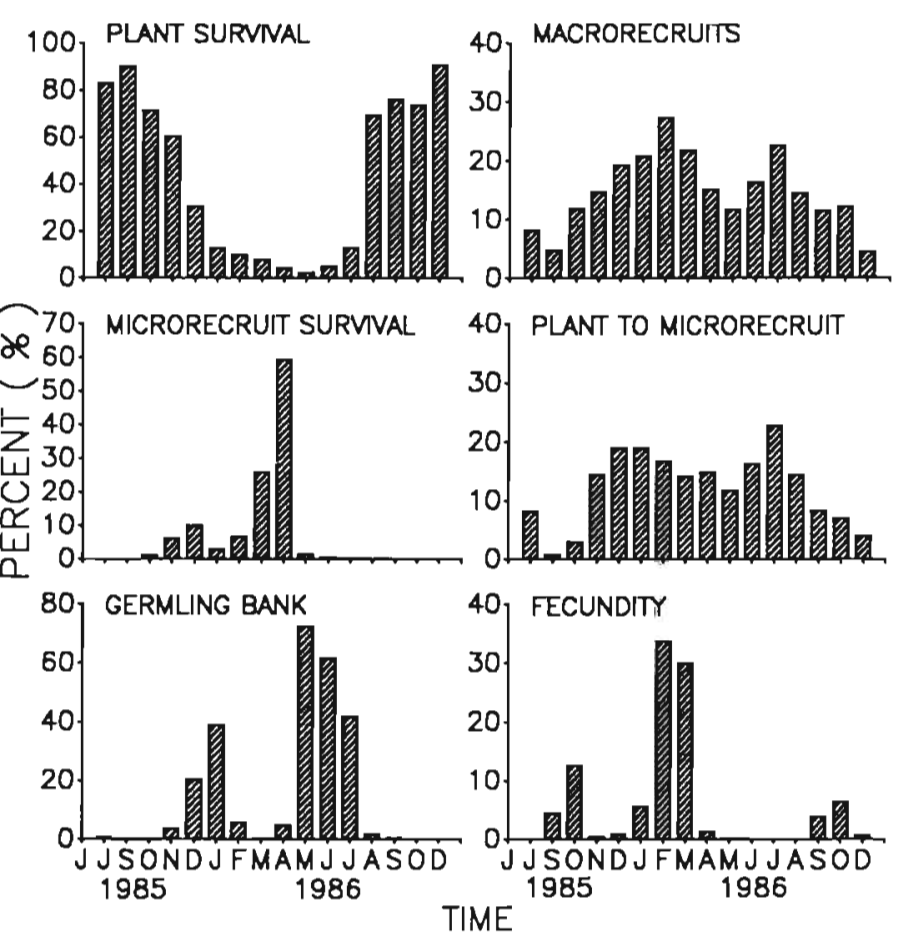

Fig. 6. Fucus distichus. Relative contribution (\%) of different matrix parameters in each yearly matrix $\mathrm{Y}$ to population growth rate, as estimated from elasticity analysis. Only the starting month of each yearly matrix is indicated in the $x$-axis. Matrix parameters are as in Fig. 4, except plant to microrecruit: transition of plants in Size Classes 1 to 6 to microrecruits

Contribution of plant survival to growth rate is mainly attributable to contributions from plants in Size Classes 2 to 4 ; contributions from the remaining size classes are $\approx<15 \%$. For the years starting from February to July 1986, the contribution of the transition from plant to microrecruits is generally the most important. In contrast, plant survival is more important at other times. This is consistent in all size classes with fecundity usually being the least important. Although plants in Size Classes 1 and 2 never became fertile, over a year's period, some of them would have grown larger and become fertile, hence, could also contribute to fecundity. However, this contribution is generally low.

Only in the July $85-J u l y ~ 86$ matrix does the stable distribution have more macro-plants than recruits. At all other times, recruits dominate. There is no clear relationship between the proportion of recruits and population growth rate, i.e., having more recruits does not guarantee a growth rate $>1$. The initial size distributions are all significantly different (chi-square test, $p<0.05$ ) from the corresponding stable distributions projected from the yearly matrices. Reproductive values for Size Classes 5 and 6 are the highest of all categories in July 85 -July 86 to January 86 -January 87 and
August 86 -August 87 to December 86 -November 87 matrices. At other times, values for Size Classes 3 and 4 are comparable or higher than those for Size Classes 5 or 6 . This suggests that all these size classes are important in contributing to future generations of the population.

\section{Simulation and projection}

The overall matrix $\mathbf{T}$ has a dominant eigenvalue $=0.6992$, indicating that the population would eventually go extinct if its growth were to be defined by the 28 mo cycle. However, if the population were to continue to grow for another year, say to follow the pattern in 1985 to 1986 ; then the population could recover and attain a positive growth rate.

Simulations using random combinations of different matrices projected over different periods of time all yielded very wide ranges of growth rates (Table 1). In the simulation using random combinations of monthly matrices projected over $5 \mathrm{yr}$, only 13 of the 100 replicates yielded a growth rate $>1$.

Seasonal matrices (Table 2) indicate a projected increase in the population during fall, and a decline during spring and summer in both 1986 and 1987. Growth during winter is variable, declining in 1986 but increasing in 1987. Simulations using random combination of seasonal matrices generally yielded a growth rate $<1$ (Table 1 ).

Several combinations of yearly matrices could be used in the simulation, e.g. July $85-$ July 86 versus July 86 -July 87 or September 85 -September 86 versus September $86-$ September 87 . The December 85-December 86 and December 86-November 87 matrices were chosen to be consistent with the time

Table 1. Fucus distichus. Results of the simulation showing the mean \pm SD, and highest and lowest growth rates $(\lambda)$ recorded from using different combinations of transition matrices projected over different fixed periods of time. The mean value is based on 100 replicates

\begin{tabular}{|lccc|}
\hline \multirow{2}{*}{ Models } & \multicolumn{3}{c|}{ Growth rate } \\
& Mean $\pm \mathrm{SD}$ & Highest & Lowest \\
\hline $\begin{array}{l}\text { Monthly matrix } \\
5 \mathrm{yr}\end{array}$ & $0.503 \pm 0.076$ & 5.800 & 0.012 \\
$\begin{array}{l}\text { Seasonal matrix } \\
5 \mathrm{yr}\end{array}$ & $1.094 \pm 0.123$ & 8.043 & 0.034 \\
$10 \mathrm{yr}$ & $0.911 \pm 0.134$ & 6.460 & 0.007 \\
$\begin{array}{l}\text { Yearly matrix } \\
5 \mathrm{yr}\end{array}$ & $0.899 \pm 0.179$ & 16.060 & 0.035 \\
$10 \mathrm{yr}$ & $1.471 \pm 0.555$ & 52.093 & 0.008 \\
\hline
\end{tabular}


Table 2. Fucus distichus. Dominant eigenvalues of the seasonal matrices

\begin{tabular}{lcc|}
\hline Season & 1986 & 1987 \\
\hline Winter & 0.6469 & 1.4984 \\
Spring & 0.6455 & 0.4646 \\
Summer & 0.5695 & 0.6894 \\
Fall & 1.7755 & 1.7423 \\
\hline
\end{tabular}

period covered by the simulations using seasonal matrices. The December 86 -November 87 matrix was further pre-multiplied with the monthly matrix for November to December 1985 in order to obtain a matrix that covers a full year. (Sampling in November 1986 was missed, hence monthly matrix for November to December 1986 was not available for use in this purpose.) Results of the simulation using random combination of these 2 December-December matrices generally yielded very low growth rates, although occasionally very high growth rates were also obtained (Table 1).

Simulations based on seasonal or yearly matrices over different fixed periods of time did not yield consistent results. Both mean growth rates $>$ or $<1$ were obtained (Table 1). In general, however, a negative growth rate was obtained in $>60 \%$ of the simulation runs.

\section{DISCUSSION}

It was not possible to get an exact fit between the sizes of the total population simulated from the models and estimated from field observations (Fig. 1). Since field observations were based on 3 quadrats, variations among samples would always be expected. The number of plants in Size Class 1 projected from the model, especially for the months from June to August 1986 , had to be made larger than that observed in order to keep the simulated number of plants in other size classes in later months as close to that observed as possible. Some of the inaccuracies in the model parameters are likely to be due to error or variation in the estimate of the number of macrorecruits, or to the lack of information on the transition of the intermediate microrecruit stages. Nevertheless, the number of individuals in these stages, though largely hypothetical, is within a realistic range indicated by the number of microrecruits on the settling blocks.

The inclusion of intermediate recruit stages (Microrecruit Stages 2 and 3) is necessary to provide the timedelay between micro- and macro-recruitments. With this time-delay, it became possible to separate and evaluate the different stages between the transition from plant reproduction to recruitment. Specifically, there are at least 2 steps in this sequence, from reproduction to microrecruits, and from microrecruits to visible macrorecruits. Size Class 1 included new macrorecruits entering the population, as well as older macrorecruits that did not grow into the next size class. It also included die-back or remnants of older plants. These categories are represented by different matrix entries, hence their relative contribution to population growth can be assessed separately. This minimizes the potential problem of having to deal with complex parameters which are the sum or product of different demographic traits.

The model is realistic within the time period sampled, and thus should be able to reveal information about the dynamics of the population within that period. However, what is equally interesting is that the models can also be used to simulate the dynamics of the population into the future. One of the inherent properties of the matrix model is time-invariance of the matrix parameters. This, as suggested by Cohen (1979), can be partly overcome by simulating the growth of the population with random combinations of matrices. This technique has been used with some success in higher plants, e.g. Arisaema triphyllum (L.) Torr. (Bierzychudek 1982), Plantago lanceolata L. (van Groenendael \& Slim 1988) and Carex bigelowii Torr. ex Schwein. (Carlsson \& Callaghan 1991).

Although sampling for this Fucus distichus population covered a period of more than $2 \mathrm{yr}$, the observed data do not suggest any recurring pattern with respect to the distribution and size of the population. Variations in matrix transition probabilities among months and between years are large, hence it appears that the population did not behave in any cyclical pattern. However, the population was starting to increase in size towards the end of the sampling period (Fig. 1). This could suggest that the sampling period represented only a fraction of the population cycle. Several cohorts within the population had been followed to their extinction (Ang 1991a, b). While the phenomena of recruitment, growth, reproduction, and death were the same for each cohort, the presence of cohorts overlapping in time could easily dampen any cyclical pattern exhibited by these biological phenomena.

Depending on the time period covered by the matrix, different assumptions are made when using random combinations of matrices to simulate population change. Random combinations of monthly matrices assume that other than a constant temporal sequence, month to month phenomena are independent from each other. Any change in the population within a month, defined by the matrix elements, is not related to changes in the previous or subsequent months. Random combinations of seasonal matrices assume a close relationship among 
months within the same season but not between seasons. Similar assumptions hold for the yearly matrices.

To represent the dynamics of a population over an extended sampling period, both the mean matrix (e.g. Werner \& Caswell 1977) and product matrix (e.g. Huenneke \& Marks 1987) have been used. In the former approach, transition values of different matrices representing different time periods are averaged. This approach assumes that variabilities among time periods are not important. In the latter approach, transition values of the matrices are multiplied. The variabilities among time periods are thus incorporated in the product.

Monthly monitoring of the individuals within the Fucus distichus population provided a very detailed information on the behaviour of this population. It indicated that growth and mortality among individuals are highly variable among months. The construction of seasonal and yearly matrices as a product, rather than as a mean, of monthly matrices is thus a more appropriate approach to account for the variabilities in the growth and survival of $F$. distichus individuals among months. These variabilities, as reflected in the matrix elements, are results of biotic interactions within the population, as well as effects of environmental variations on the population over time (Ang 1991a, b, Ang \& De Wreede 1992)

A matrix model summarizes the information about current population behaviour. Despite the constraints of being deterministic in its prediction, considerable information about the population can be inferred from the analysis. Fucus distichus plants do not exhibit any vegetative reproduction, hence recruitment is essential for the continuity of the population. Positive growth (eigenvalues $>1$ ) for the population can only be attained in the presence of reproduction and recruitment. This is in contrast to some other fucoids like Sargassum siliquosum where results from an elasticity analysis indicate that vegetative regeneration from the holdfast is far more important to population growth than does recruitment, and that the contribution of recruitment to population growth is almost close to 0 (Ang \& De Wreede 1990). In general, however, recruitment contributes to the stability for instability) and growth of marine benthic populations. The significance of this contribution has increasingly been recognized in recent years both in algal (e.g. Reed et al. 1988, Nisbet \& Bence 1989) and animal populations (e.g. Roughgarden et al. 1988).

The reduction in the relative importance of plant survival to population growth rate indicated by the yearly matrices is compensated for by the increased importance of other parameters such as the survival of macrorecruits to juvenile and reproductive size classes and the contribution of plant reproduction to micro- recruit stages. These parameters, however, actually represent the contribution of new individuals that entered the population over the course of a year. The relative importance of plant survival measured by the elasticity analyses represents mainly the contribution of the older plants that existed at the start of the period. These plants suffered increased mortality, hence, their contribution to the population growth rate also decreased over time.

A large contribution of plant survival to population growth rate is characteristic of long-lived plants like trees (Hartshorn 1975, Enright \& Ogden 1979). This suggests that even in the absence of any recruitment, the decline of the population would not be abrupt. In such a case, the chances are high that at some time the population may recover from a continuous decline as a result of a sudden pulse of recruits.

In the simulation, each month, season or year, was chosen from 2 matrices, each having an equal probability of being included in each run. One of the 2 matrices (the 'good' matrix) projects a higher growth rate, thus depicts a 'good' month, season or year, than the other (the 'bad' matrix). An extremely high growth rate could be obtained, or vice versa, if the 'good' or the 'bad' matrix is continuously being chosen over the period simulated. In reality, a 'good' or 'bad' month, season, or year described respectively by the 'good' or 'bad' matrix may not recur over a prolonged period. These extreme growth rates are therefore very unlikely.

It can not be ascertained which of the 3 ways of randomly combining matrices used in the simulation is more realistic. This needs to be tested with a longer term of sampling, i.e. beyond the 2 yr covered by this study. Nevertheless, while this Fucus distichus population is declining, it may recover in the future. The population is probably being maintained by an occasional pulse of a large number of recruits. The year 1986 may be an example of such a good year.

On a monthly basis, the presence of a germling bank had little effect on population growth rate. However, if there were no germling bank for the whole year, the effect could be more significant. None of the yearly matrices showed a growth rate $>1$ if the probability of microrecruits being present in the germling bank was set to 0 . The germling bank appears to compensate for the lack of fecundity, as indicated by a negative relationship between the contributions of fecundity and the germling bank to growth rate. The seed bank of higher plants has a stabilizing effect on population growth rate (Schmidt \& Lawlor 1983). The germling bank in algae, if it exists, is likely to play a similar role. How significant this role is, to this Fucus distichus as well as to other algal populations, remains to be shown in the field (Santelices 1990). 
Many algae produce a large number of propagules (Hoffmann 1987. Santelices 1990), but only a small fraction of this is recruited into the population. An even smaller fraction of this ever survives and becomes large enough to contribute to future reproduction. Hence, a stable distribution with a large proportion of the plants in the recruit stage is probably typical of many algae. This is not true for another brown alga Sargassum siliquosum where the proportion of recruits at the stable distribution is small (Ang \& De Wreede 1990). However, in S. siliquosum, new laterals regenerate from the holdfast every year. As noted earlier, this mode of vegetative reproduction is far more important than sexual reproduction to population growth of S. siliquosum.

Current size distribution is a reflection of past events. Stable distributions calculated from the monthly and yearly matrices are different from the current size distribution of the Fucus distichus population. The existence of a stable distribution hinges on the presence of a constant growth rate in the population. But, as Bierzychudek (1982) argued, the attainment of a constant growth rate is based on the assumption of the existence of a constant environment. In reality, the environment is never constant and a constant growth rate, as well as a stable distribution, will never be attained. A population may therefore be much more influenced by its size structure than by its growth rate. This argument may be particularly relevant to this Fucus distichus population. If the population were being maintained by occasional influxes of recruits, as suggested earlier, then the influx could serve as a disturbance that keeps the population in a non-equilibrium state. The existence of a large number of recruits in this population, especially those as Stages 2 and 3 microrecruits, remains to be verified. Further analyses incorporating these stages should prove to be interesting in elucidating how the size distribution is being structured and the possible influence of the environment on the current size structure of this population.

Low reproductive values for recruits and Size Classes 1 and 2 are expected as they do not contribute directly to fecundity. For the other 4 size classes, a positive relationship between reproductive values and plant size classes is discernible. Although the number of microrecruits originating from each reproductive plant may be the same, i.e. the actual number of eggs produced per plant may be independent of plant size, but because the probability of being reproductive is higher for larger plants, there is therefore a positive relationship between fecundity value and plant size, hence between reproductive values and plant size classes. However, there is no theoretical basis to assume that reproductive value is always positively related to plant size class. There are examples in higher plants where this relationship does not hold (e.g. Carex bigelowii, Carlsson \& Callaghan 1991).

The life span of Fucus distichus is about 2 to $3 \mathrm{yr}$ (Ang 1991a, b). With a high mortality of the recruits, iteroparity should be favoured (Charnov \& Schaffer 1973). Iteroparity in $F$. distichus appears to be achieved by the formation of receptacles at different terminal branches at different times (Ang 1992). This enables other non-reproductive terminal branches of the plant to continue to grow, thus minimizing the cost of reproduction (Ang 1992). This must have a significant advantage for $F$. distichus. Reduction in mortality rate and increase in plant size both increase the importance of the plant to population growth rate, the latter through an increase in reproductive value, and hence increased contribution to recruitment.

The matrix models developed here were based on the size structure of the Fucus distichus population. Given that both age and size are significant state variables in describing the demographic parameters of this population (Ang 1991a, b), further improvement of the models may include age as an additional state variable. Density-dependent survivorship and stochasticity of recruitment are probably two important factors that control the outcome of any population change. Variation within the population has not been accounted for in this study. The information on population dynamics was based on mean data from three quadrats. It is possible that spatial heterogeneity may allow patches of the population to exhibit different dynamics. If this is true, then there could be variation in the dispersal of propagules resulting in spatial heterogeneity in recruitment. This should make the study of Fucus distichus in particular, and algal population dynamics in general, even more challenging.

Acknowledgements. This study was partially funded by Natural Science and Engineering Research Council of Canada grant no. 5-89872 to R. De Wreede. Critical comments from P. G. Harrison, R. Santos, G. Sharp, R. Turkington and the anonymous reviewers greatly improved the earlier drafts of the manuscript. P.O.A. held a University of British Columbia (UBC) graduate fellowship, an International Centre for Ocean Development fellowship, an Edith Asthon Memorial Scholarship (UBC), and a Kit Malkin Scholarship (UBC) during the duration of this study. The revision of the manuscript was undertaken during P.O.A.'s tenure as a postdoctoral fellow at the Halifax Fisheries Research Laboratory, Fisheries and Oceans Canada.

\section{LITERATURE CITED}

Áberg. P. (1992a). A demographic study of two populations of the seaweed Ascophyllum nodosum. Ecology 73: 1473-1487

Aberg, P. (1992b). Size-based demography of the seaweed Ascophyllum nodosum in stochastic environments. Ecology 73: $1488-1501$ 
Anderson, B. N. (1974). A mathematical model for the growth of giant kelp. Simulation 22: 97-106

Ang, P. O. Jr (1987). Use of projection matrix models in the assessment of harvesting strategies for Sargassum. Hydrobiologia 151/152: 335-339

Ang, P. O. Ir (1991a). Natural dynamics of a Fucus distichus (Phaeophyceae, Fucales) population: reproduction and recruitment. Mar. Ecol. Prog. Ser. 78: 71-85

Ang, P. O. Jr (1991b). Age- and size-dependent growth and mortality in a population of Fucus distichus. Mar. Ecol. Prog. Ser. 78: 173-187

Ang, P. O. Jr (1991c). Natural dynamics and matrix models of a Fucus distichus (Phaeophyceae, Fucales) population in Vancouver, British Columbia, Canada. Ph.D. thesis, University of British Columbia, Vancouver

Ang, P. O. Jr (1992). Cost of reproduction in Fucus distichus. Mar. Ecol. Prog. Ser. 89: 25-35

Ang, P. O. Jr, De Wreede, R. E. (1990). Matrix models of algal life history stages. Mar. Ecol. Prog. Ser. 59: 171-181

Ang, P. O. Jr, De Wreede, R. E. (1992). Density-dependence in a population of Fucus distichus. Mar. Ecol. Prog. Ser. 90: $169-181$

Ang, P. O. Jr, De Wreede R. E., Shaughnessy F., Dyk, L. (1990). A simulation model of an Iridaea splendens (Gigartinaceae, Rhodophyta) population in Vancouver. Canada. Hydrobiologia 204/205: 191-196

Bierzychudek, P. (1982). The demography of Jack-in-thepulpit, a forest perennial that changes sex. Ecol. Monogr. 52: $335-351$

Bold, H. C., Wynne, M. J. (1985). Introduction to the algae, 2nd edn. Prentice-Hall, Englewood Cliffs

Burgman, M. A., Gerard, V. A. (1990). A stage-structured, stochastic population model for the giant kelp Macrocystis pyrifera. Mar. Biol. 105: 15-23

Carlsson, B. A., Callaghan, $\Upsilon$ V. (1991). Simulation of fluctuating populations of Carex bigelowii tillers classified by type, age and size. Oikos 60: 231-240

Caswell, H. (1989). Matrix population models. Sinauer Associates Inc., Sunderland, MA

Charnov, E. L., Schaffer, W. M. (1973). Life history consequences of natural selection: Cole's results revisited. Am Nat. 107: 791-793

Cohen, J. E. (1979). Comparative statics and stochastic dynamics of age-structured populations. Theor. Populat. Biol. 16: 159-171

de Kroon, H., Plaisier, A., van Groenendael, J., Caswell, H. (1986). Elasticity: the relative contribution of demographic parameters to population growth. Ecology 67: $1427-1431$

De Wreede, R. E. (1986). Demographic characteristics of Pterygophora californica (Laminariales, Phaeophyta). Phycologia 25: 11-17

This article was submitted to the editor
Enright, N., Ogden, J. (1979). Applications of transitional matrix models in forest dynamics: Araucaria in Papua New Guinea and Nothofagus in New Zealand. Aust. J. Ecol. 4: 3-23

Hartshorn, G. S. (1975). A matrix model of tree population dynamics. In: Golley, F. B., Medina, E. (eds.) Tropical ecological systems. Springer-Verlag, New York, p. 41-51

Hoffmann, A. J. (1987). The arrival of seaweed propagules at the shore: a review. Botanica mar. 30: 151-165

Huenneke, L. F., Marks, P. L. (1987). Stem dynamics of the shrub Alnus incana spp. rugosa: transition matrix models. Ecology 68: 1234-1242

Jackson, G. A. (1987). Modelling the growth and harvest yield of the giant kelp Macrocystis pyrifera. Mar. Biol. 95: $611-624$

Jackson, G. A., James, D. E., North, W. J. (1985). Morphological relationship among fronds of the giant kelp Macrocystis pyrifera off La Jolla, California. Mar. Ecol. Prog. Ser. 26: 261-270

Leslie, P. H. (1945). On the use of matrices in certain population mathematics. Biometrika 33: 183-212

Leslie, P. H. (1948). Some further notes on the use of matrices in population mathematics. Biometrika 35: 213-245

Nisbet, R. M., Bence, J. R. (1989). Alternative dynamic regimes for canopy-forming kelp: a variant on densityvague population regulation. Am. Nat. 134:377-408

Reed, D. C., Laur, D. R., Ebeling, A. W. (1988). Variation in algal dispersal and recruitment: the importance of episodic events. Ecol. Monogr. 58: 321-335

Roughgarden, J., Gaines, S., Possingham, H. (1988). Recruitment dynamics in complex life cycles. Science 241: $1460-1466$

Santelices, B. (1990). Patterns of reproduction, dispersal and recruitment in seaweeds. Oceanogr. mar. Biol. A. Rev. 28: $177-276$

Schmidt, K. P., Lawlor, L. R. (1983). Growth rate projection and life history sensitivity for annual plants with a seed bank. Am. Nat. 121: 525-539

Seip, K. L. (1980). A computational model for growth and harvesting of marine alga Ascophyllum nodosum. Ecol. Modelling 8: 189-199

Silverthorne, W. (1977). Optimal production from a seaweed resource. Botanica mar. 20: 75-98

Smith, B. D. (1986). Implications of population dynamics and interspecific competition for harvest management of the seaweed Laminaria. Mar. Ecol. Prog. Ser. 33: 7-18

van Groenendael, J. M., Slim, P. (1988). The contrasting dynamics of two populations of Plantago lanceolata classified by age and size. J. Ecol. 76: 585-599

Werner, P. A., Caswell, H. (1977). Population growth rates and age vs. stage distribution models for teasel (Dipsacus sylvestris Huds.). Ecology 58: 1103-1111

Manuscript firsi received: November 11, 1991

Revised version accepted: January 13, 1993 